\title{
Uncovering the secretes of allergic inflammation
}

\author{
Michael Brusilovsky, ${ }^{1,2}$ Mark Rochman, ${ }^{1,2}$ Nurit P. Azouz, ${ }^{1,2}$ Lydia E. Mack, ${ }^{1,2}$ and Marc E. Rothenberg,2 \\ 'Division of Allergy and Immunology, Cincinnati Children's Hospital Medical Center, Cincinnati, Ohio, USA. ²Department of Pediatrics, University of Cincinnati College of Medicine, Cincinnati, Ohio.
}

\begin{abstract}
Allergic asthma is a chronic inflammatory lung disease associated with increased cytokine secretion. Aspects of airway inflammation are also linked to a common genetic variant that corresponds to the small GTPase, Rab27, a protein involved in vesicular trafficking in immune cells. However the mechanisms by which Rab27 contributes to airway inflammation and cytokine release remain ambiguous. In this issue of the $J C l$, Okunishi et al. explored the role that the Rab27 effector, exophilin-5, has in allergic inflammation. Exophilin-5-deficient mice and asthma mouse models revealed that exophilin- 5 regulates IL-33 production and the Th2 response. Notably, exophilin-5 deletion enhanced IL-33 release and pathogenic Th2 responsiveness through the mTOR pathway and altered intracellular IL-33 trafficking. This work provides insights into the molecular mechanisms that underlie inflammatory lung disease.
\end{abstract}

\section{Vesicular trafficking}

The endoplasmic reticulum and the Golgi comprise the two main cellular organelles involved in conventional vesicular trafficking. Vesicular carriers mediate a continuous flux of newly synthesized and secreted proteins between these compartments (1). However, it has recently been shown that many proteins use unconventional routes, including autophagy, cellular stress, membrane hyperpermeabilization, and pore formation to control protein trafficking (2). Moreover, some proteins, such as the IL-1 superfamily of cytokines (e.g., IL-1 $\beta$ ) may use any of the above pathways, depending upon the secretory stimuli (2).

\section{Chronic inflammatory lung disease}

Allergic asthma is a chronic inflammatory lung disease associated with the marked secretion of a series of cytokines associ- ated with type $2 \mathrm{~T}$ cell responses (type 2 immunity) (3); among these cytokines, recent attention has focused on IL-33, an epithelial and endothelial cytokine that potently activates inflammatory cells bearing its receptor (designated ST2) including mast cells, eosinophils, and $\mathrm{T}$ cells (4). IL-33 is a unique cytokine in that it is an alarmin, also known as a danger signal molecule, stored in the nucleus, where it is bound to histone proteins via its chromatin binding domain. Although a direct nuclear function for IL-33 has not yet been identified, its tight interaction with chromatin regulates its activity and release (5). It is appreciated that IL-33 is released following cellular injury $(6,7)$, but it remains unclear whether IL-33 is actively secreted or uses unconventional secretory pathways, such as pore formation or autophagosomes. As such, the secretory mechanisms of IL-33 release remain to be determined.

\section{Related Article: p. 3919}

Conflict of interest: MER is a consultant for Pulm One, Spoon Guru, ClostraBio, Serpin Pharm, Astra Zeneca, and Guidepoint, and has an equity interest in the first four companies listed as well as royalties from reslizumab (Teva Pharmaceuticals), PEESSv2 (Mapi Research Trust), and UpToDate. MER is an inventor on patents owned by Cincin nati Children's Hospital. MER has received research support from Adare Pharmaceuticals, Regeneron, Allakos, and Astra Zeneca. NPA is an inventor on patents 62/126,814 and 63/017,027, owned by Cincinnati Children's Hospital. Copyright: () 2020, American Society for Clinical Investigation.

Reference information: J Clin Invest. 2020;130(7):3419-3421. https://doi.org/10.1172/JCI138343.

\section{A synaptotagmin-like protein that regulates secretion}

Rab27a is a ubiquitously expressed intracellular protein that is one of 70 members of the Rab family of proteins, which in turn is a member of the Ras superfamily of small $G$ proteins. Rab proteins generally possess GTPase activity and regulate many steps involved in membrane trafficking, including vesicle formation, vesicle movement along actin and tubulin networks, and membrane fusion. These processes are particularly important for the trafficking and recycling of cell surface proteins from the Golgi to the plasma membrane. Rab27a regulates important secretionmachinery functions in mast cells and dendritic and epithelial cells (8), all of which have essential roles in antigen-driven allergic immune responses. The importance of Rab27a in human disease is highlighted by its recessive deficiency causing Griscelli syndrome, an immunodeficiency syndrome associated with hemophagocytic lymphohistiocytosis (9). There are at least 11 known Rab27a accessory proteins that regulate its function. Among these proteins is exophilin-5, a synaptotagmin-like protein that has been reported to positively regulate secretion $(8,10)$.

\section{Exaggerated allergic inflammation}

In this issue of the JCI, Okunishi et al. report that mice deficient in exophilin-5 showed exaggerated allergic inflammation mediated by enhanced IL-33 production (11). Epithelial cells were responsible for producing increased IL-33 levels and triggering subsequent type 2 cytokine responses via Th2 cells. Furthermore, the researchers presented evidence that the IL-33/Th2 mechanism depended on mammalian target of rapamycin (mTOR), a lysosome-associated serine/threonine kinase that regulates protein synthesis, autophagy, cellular trafficking, and protein secretion (12).

Okunishi et al. reported that alveolar epithelial cells expressed high levels of exophilin-5 and that exophilin-5-defi- 


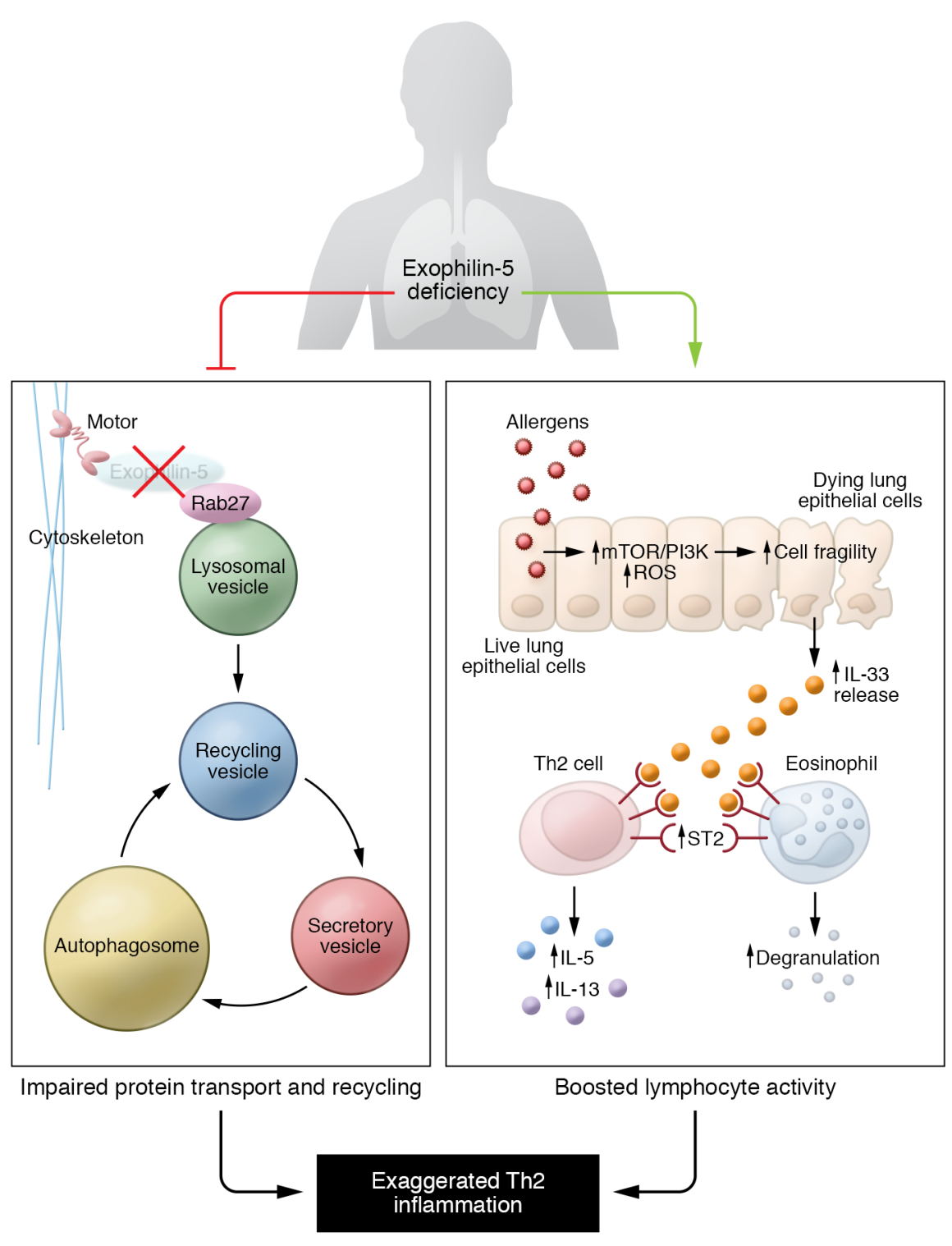

cient mice were more vulnerable to allergen challenge with Alternaria alternata (11). Indeed, A. alternata administration enhanced IL-33 release in the absence of exophilin-5, resulting in augmented airway inflammation. However, it remains unclear whether exophilin-5 directly regulates IL-33 secretion via unconventional pathways involving lysosomal trafficking, or whether exophilin-5 deficiency makes epithelial cells more susceptible to $A$. alternata-induced cellular injury and thus able to release more IL-33. It is likely that both cellular trafficking and epithelial cell fragility are involved. It is thus possible that in exophilin-5-deficient mice, epithelial cells with enhanced sensitivity to necrotic or apoptotic cell death mediate IL-33 release. Indeed, lysosomal trafficking is involved
Figure 1. Model for exophilin-5 regulation of cellular traffic and secretion mechanisms and type 2 allergic immunity. Exophilin- 5 has a central regulatory role in controlling the cellular traffic of vesicles, as well as impairing protein transport and recycling and IL-33 production. Exophilin-5 deficiency terminates vesicular trafficking mechanisms and disrupts protein recycling, autophagy, and secretion. Exophilin-5 deficiency also enhances mTOR/PIK3 signaling and ROS production. These intrinsic processes result in IL-33 accumulation and enhanced lung epithelial cell fragility, respectively, followed by IL-33 release from damaged cells. Enhanced ST2 expression in exophilin-5-deficient effector $T$ cells and eosinophils induces proatopic cytokine release and immune cell cytotoxicity and degranulation. Taken together, these processes lead to exaggerated type 2 immunity.

ciated caspase- 1 cleaves precursor IL-33 to release the active cytokine into the interstitial space (19). Thus, Okunishi et al. have provided evidence for lysosomes having a role in sensitizing cells to proapoptotic or necrotic stimuli and the consequent release of IL-33 (Figure 1 and ref. 11).

Interestingly, the mTOR activation associated with exophilin-5 deficiency can reduce overall protein degradation by the ubiquitin proteasome pathway (20). It is also notable that IL-33 is highly sensitive to proteasomal degradation $(21,22)$. Thus, inhibition of the proteasome pathway by mTOR may result in IL-33 accumulation and the subsequent release of high cytokine levels following the A. alternatainduced epithelial injury. Furthermore, Okunishi and colleagues showed that exophilin-5 deficiency and mTOR pathway amplification were associated with ST2 accumulation and enhanced proinflammatory cytokine production in Th2 cells (11). Hence, it is possible that exophilin-5 deficiency reduces the recycling rate of membrane ST2 and increases the accumulation of IL-5 and IL-13 through mTORand Rab27a-dependent mechanisms.

\section{Conclusions}

The IL-33/ST2 axis is now considered to have a pivotal role in type 2 allergic inflammation, but the mechanisms of IL-33 secretion have remained obscure. Taken together, the results of Okunishi et al. provided insights into the functions of exophilin-5 in immune secretory pathways, with a focus on the IL-33/ST2 axis (11). Okunishi et al. suggest that exophilin- 5 orchestrates 
allergic inflammatory responses in which its deficiency enhances IL-33 release and boosts ST2-dependent IL-5 and IL-13 production by Th2 cells via cellular trafficking and secretory mechanisms (Figure 1 and ref. 11). These findings uncover early secretes of the IL-33 secretory mechanisms, bringing the lysosomal protein degradation machinery and autophagy into the center stage for the development of type 2 allergic inflammation (Figure 1).

\section{Acknowledgments}

The authors thank Shawna Hottinger for medical editing.

Address correspondence to: Marc Rothenberg, Division of Allergy and Immunology, Cincinnati Children's Hospital Medical Center, MLC 7028, 3333 Burnet Avenue, Cincinnati, Ohio 45229-3039, USA. Phone: 513.636.7210; Email: rothenberg@ cchmc.org.

\section{Lee MC, Miller EA, Goldberg J, Orci L, Schek-} man R. Bi-directional protein transport between the ER and Golgi. Annu Rev Cell Dev Biol. 2004;20:87-123.

2. Rabouille C. Pathways of unconventional protein secretion. Trends Cell Biol. 2017;27(3):230-240.

3. Holt PG, Macaubas C, Stumbles PA, Sly PD. The role of allergy in the development of asthma Nature. 1999;402(6760 suppl):B12-B17.

4. Cherry WB, Yoon J, Bartemes KR, Iijima K, Kita H. A novel IL-1 family cytokine, IL-33, potently activates human eosinophils. J Allergy Clin Immunol. 2008;121(6):1484-1490.

5. Travers J, et al. Chromatin regulates IL-33 release and extracellular cytokine activity. Nat Commun. 2018;9(1):3244.

6. Garlanda C, Dinarello CA, Mantovani A. The interleukin-1 family: back to the future. Immunity. 2013;39(6):1003-1018

7. Kouzaki H, Iijima K, Kobayashi T, O'Grady SM, Kita $\mathrm{H}$. The danger signal, extracellular ATP, is a sensor for an airborne allergen and triggers IL-33 release and innate Th2-type responses. J Immunol. 2011;186(7):4375-4387.

8. Gomi H, Mori K, Itohara S, Izumi T. Rab27b is expressed in a wide range of exocytic cells and involved in the delivery of secretory granules near the plasma membrane. Mol Biol Cell. 2007;18(11):4377-4386.

9. Ménasché G, et al. Mutations in RAB27A cause Griscelli syndrome associated with haemophagocytic syndrome. Nat Genet. 2000;25(2):173-176.

10. Ostrowski M, et al. Rab27a and Rab27b control different steps of the exosome secretion pathway. Nat Cell Biol. 2010;12(1):19-30.

11. Okunishi K, et al. Exophilin-5 regulates allergic airway inflammation by controlling IL-33mediated Th2 responses. J Clin Invest. 2020;130(7):3919-3935.

12. Betz C, Hall MN. Where is mTOR and what is it doing there? J Cell Biol. 2013;203(4):563-574.

13. Ferri KF, Kroemer G. Organelle-specific ini- tiation of cell death pathways. Nat Cell Biol. 2001;3(11):E255-E263.

14. Hishita T, et al. Caspase- 3 activation by lysosomal enzymes in cytochrome c-independent apoptosis in myelodysplastic syndrome-derived cell line P39. Cancer Res. 2001;61(7):2878-2884.

15. Morgan MJ, Kim YS, Liu ZG. TNFalpha and reactive oxygen species in necrotic cell death. Cell Res. 2008;18(3):343-349.

16. Zhou R, Yazdi AS, Menu P, Tschopp J. A role for mitochondria in NLRP3 inflammasome activation. Nature. 2011;469(7329):221-225.

17. Davis MJ, Swanson JA. Technical advance: Caspase- 1 activation and IL- $1 \beta$ release correlate with the degree of lysosome damage, as illustrated by a novel imaging method to quantify phagolysosome damage. J Leukoc Biol. 2010;88(4):813-822.

18. Amaral EP, et al. Lysosomal cathepsin release is required for NLRP3-inflammasome activation by Mycobacterium tuberculosis in infected macrophages. Front Immunol. 2018;9:1427.

19. Dinarello CA. An IL-1 family member requires caspase-1 processing and signals through the ST2 receptor. Immunity. 2005;23(5):461-462.

20. Zhao J, Zhai B, Gygi SP, Goldberg AL. mTOR inhibition activates overall protein degradation by the ubiquitin proteasome system as well as by autophagy. Proc Natl Acad Sci U S A. 2015;112(52):15790-15797.

21. Lüthi AU, et al. Suppression of interleukin-33 bioactivity through proteolysis by apoptotic caspases. Immunity. 2009;31(1):84-98.

22. Zhang Y, et al. Coordinated regulation of protein synthesis and degradation by mTORC1. Nature. 2014;513(7518):440-443. 